Special issue of the 2nd International Conference on Computational and Experimental Science and Engineering (ICCESEN 2015)

\title{
State-Space Modeling of an EPW in Discrete Time and an Observer Design for State Variable Estimation
}

\author{
A. ÖZDEMIR, Z. ERDEM* AND I. USUMAN
}

Sakarya University, Department of Electrical and Electronics Engineering, Sakarya, Turkey

\begin{abstract}
The purpose of this study is to obtain the dynamic model of an electrical powered wheelchair and to estimate the state variables of right and left DC motor currents with the designed observer. First, the dynamic equations are written and then discrete-time state space model of the electrical powered wheelchair is directly obtained from this dynamic equations. Discrete time state space model of the electrical powered wheelchair is verified with the transfer function obtained using the dynamic equations. In addition, the accuracy of the estimated left and right DC motor current values are validated in the simulation results.
\end{abstract}

DOI: $10.12693 /$ APhysPolA.130.228

PACS/topics: 02.30.Yy, 89.20.Kk, 02.30.Cj, 02.70.-c, 07.05.Dz

\section{Introduction}

According to the World Health Organization (WHO), $10 \%$ of the population worldwide suffer from some physical disability [1]. Robotics technology has grown very fast with the advancement of navigation, control systems, and sensors technologies. This technologies can be applied to electrical powered wheelchairs (EPWs), which provide functional mobility for people with both lower and upper extremity impairments. EPW control technology under adverse driving conditions should be improved to provide mobility and comfort for EPW users. Various control methods of EPWs are found in literature [2-7], which are concerned with velocity control, traction control, suspension control, stability control, stair-climbing, and wheelchair navigation.

The most commonly controlled variable in an EPW is speed. During normal operation, the wheelchair driver applies command inputs using a joystick or similar device, based on their perception of the wheelchair's speed and direction [8]. For the speed control of the EPWs, the state variables of the model have to be measured or estimated. In some conditions, measurement of some state variables could be difficult. Therefore, in this paper, the dynamic model of a wheelchair and an observer design for current estimation of the right and left DC motors are investigated. In this paper, the EPW has two wheels and both wheels are driven separately by DC motors, placed on one axis. Both these wheels serve for driving and steering of the wheelchair. As a counterweight, the EPW is equipped also with two castor wheels that are placed in the front of the wheelchair.

Modeling is stated as identifying the principal physical dynamic effects of analyzed system by considering the general conservative laws of the discipline of the interest and by deriving the differential equations for describing

\footnotetext{
*corresponding author; e-mail: zekiye@sakarya.edu.tr
}

the dynamical behavior of the system [8]. The MIMO description of EPW dynamics leads to observation of the linear and angular velocities of right and left wheels, which are interacting with each other [9].

Although the transfer function and/or the approximate models are generally used in literature for modeling of the EPW $[2-4,6,8,9]$, as a contribution to the literature, in this study the discrete time state space model of the EPW is directly obtained from the differential and algebraic equations of the relevant discipline.

This paper is organized as follows; Sect. 2 gives an overview about the equation of state space modeling in discrete time of EPW, presents the system structure and shows the mathematical representation of the wheelchair dynamic model and the observer design for wheelchair system for the state variables. Section 3 shows the simulation results of the state space model validation and the estimated state variables via the designed observer model. Section 4 summarizes and concludes the paper.

\section{State space modeling of EPW}

The EPW analyzed in this paper, has two electrical powered rear wheels and two passive front castor wheels. Each of the rear wheels is independently driven by a similar DC powered motor. Using the difference between kinetic and potential energies of the wheelchair, Lagrange formalism is used to derive the general set of differential equations that describe the time evolution of powered wheelchair.

The model of EPW is shown in Fig. 1. "O" is the center of gravity of EPW. In this model $\theta_{r}$ and $\theta_{l}$ are angular velocities of left and right wheels, $\phi$ describes the direction of motion, $R_{1}$ is the radius of rear wheel, $L$ is the length between two wheels of the EPW and $X$ vector denotes the variables of the directions. The state variables of the EPW are given below as a vector matrix form;

$$
X=[x y \phi]^{T}, \quad \theta=\left[\theta_{r} \theta_{l}\right]^{T} .
$$

Total kinetic energy for the rotational and linear motion of the EPW is given in Eq. (1). 


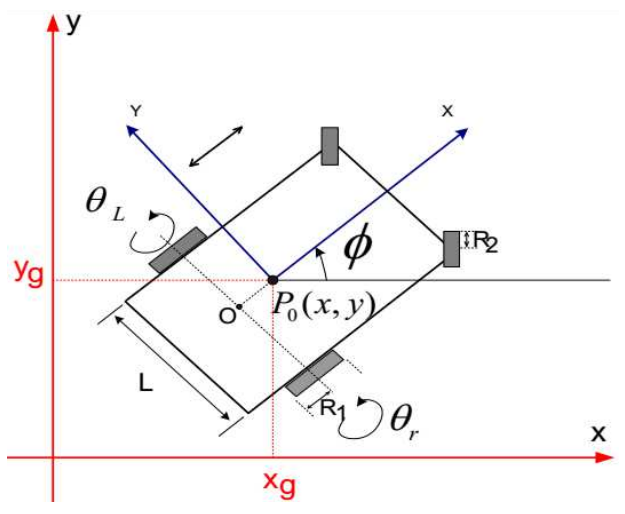

Fig. 1. Model of EPW.

$$
\begin{aligned}
K= & K_{\text {linear }}+K_{\text {rotation }}+K_{\mathrm{r}}+K_{\mathrm{l}}, \\
K & =\underbrace{\frac{1}{2} m\left(\frac{R_{1}}{2}\left(\dot{\theta}_{r}+\dot{\theta}_{l}\right)\right)^{2}}_{\text {Translational movement energy of vehicle }} \\
& +\underbrace{\frac{1}{2} J\left(\frac{R_{1}}{L}\left(\dot{\theta}_{r}-\dot{\theta}_{l}\right)\right)^{2}}_{\text {Rotation energy of vehicle }}+\underbrace{\frac{1}{2} J_{w}\left(\dot{\theta}_{r}^{2}+\dot{\theta}_{l}^{2}\right)}_{\text {Rotation energy of wheel }}
\end{aligned}
$$

The torques $\left(T_{r}, T_{l}\right)$ denote the moment of inertia produced by the wheels on the left and right sides of the EPW respectively. Equivalent torque matrix is obtained using Lagrange equation as represented in Eq. (2):

$$
\begin{aligned}
& {\left[\begin{array}{c}
T_{r}(t) \\
T_{l}(t)
\end{array}\right]=\left[\begin{array}{c}
\frac{m R_{1}^{2}}{4}+J w+\frac{J R_{1}^{2}}{w^{2}} \frac{m R_{1}^{2}}{4}-\frac{J R_{1}^{2}}{w^{2}} \\
\frac{m R_{1}^{2}}{4}-\frac{J R_{1}^{2}}{w^{2}} \frac{m R_{1}^{2}}{4}+J w+\frac{J R_{1}^{2}}{w^{2}}
\end{array}\right]} \\
& \times\left[\begin{array}{c}
\frac{\mathrm{d} w_{r}(t)}{\mathrm{d} t} \\
\frac{\mathrm{d} w_{L}(t)}{\mathrm{d} t}
\end{array}\right] .
\end{aligned}
$$

Electrical moment produced by right and left DC motors is given below;

$$
\begin{aligned}
& T_{\mathrm{e} i}(t)=K_{t i} i_{a i}(t), \quad i=\mathrm{r}, 1, \\
& T_{\mathrm{m} i}(t)=J_{\mathrm{m} i} \frac{\mathrm{d} w_{\mathrm{m} i}(t)}{\mathrm{d} t}+B_{\mathrm{m} i} w_{\mathrm{m} i}(t) \\
& \quad+N\left(T_{i}(t)+T_{\mathrm{Load} i}(t)\right), \quad i=\mathrm{r}, \mathrm{l} .
\end{aligned}
$$

The descriptions of the EPW parameters are given in Table I.

The electrical moments produced by DC motors must be greater then $T_{r}$ and $T_{l}$, the mechanical moments

\begin{tabular}{|c|c|}
\hline$\overline{\text { Symbol }}$ & Description \\
\hline$m$ & Total mass for person on board and wheelchair body [kg] \\
\hline$R_{1}$ & Radius of rear wheels $[\mathrm{m}]$ \\
\hline$J_{w}$ & Moment of inertia of the rear wheel $\left[\mathrm{kg} \mathrm{m}^{2}\right]$ \\
\hline$J$ & Total moment of inertia with the total mass $\left[\mathrm{kg} \mathrm{m}^{2}\right]$ \\
\hline$L$ & Distance between rear wheels $[\mathrm{m}]$ \\
\hline$W_{i}$ & Angular velocity of right wheel DC motor $[\mathrm{rad} / \mathrm{s}] i=\mathrm{r}, \mathrm{l}$ \\
\hline$T_{i}$ & Moment of inertia of right wheel DC motor $\left[\mathrm{kg} \mathrm{m}^{2}\right] i=\mathrm{r}, 1$ \\
\hline$T_{\mathrm{e} i}$ & Electrical moment of DC motor $i=\mathrm{r}, 1$ \\
\hline$T_{\mathrm{m} i}$ & Mechanical moment of DC motor $i=\mathrm{r}, \mathrm{l}$ \\
\hline$B_{m}$ & Friction in the DC motor $[\mathrm{N} \mathrm{ms}]$ \\
\hline$L_{a i}$ & DC motor inductance $[\mathrm{H}] i=\mathrm{r}, \mathrm{l}$ \\
\hline$R_{a i}$ & DC motor resistance $[\mathrm{Ohm}] i=\mathrm{r}, \mathrm{l}$ \\
\hline$i_{a i}$ & DC motor rotor side current $i=\mathrm{r}, \mathrm{l}$ \\
\hline$N$ & Gear ratio \\
\hline$T$ & Sampling time $[\mathrm{s}]$ \\
\hline \multicolumn{2}{|r|}{$K_{t l} i_{a l}(t)-B_{m l} W_{m l}(t)-N T_{\text {Load } l}(t)=$} \\
\hline & $\left(J_{m l}+N^{2}\left(\frac{m R_{1}^{2}}{4}+J_{w}+\frac{J R_{1}^{2}}{W^{2}}\right)\right) \frac{\mathrm{d} W_{m l}(t)}{\mathrm{d} t}$ \\
\hline & $-N^{2}\left(\frac{m R_{1}^{2}}{4}-\frac{J R_{1}^{2}}{W^{2}}\right) \frac{\mathrm{d} W_{m r}(t)}{\mathrm{d} t}$ \\
\hline
\end{tabular}
applied to the motor shafts. Electrical moments are equated to the mechanical moments in matrix (2), and Eqs. (3), (4) to obtain the continuous time state space model of the EPW.

$$
\begin{aligned}
& K_{t r} i_{a r}(t)-B_{m r} W_{m r}(t)-N T_{\text {Load } r}(t)= \\
& \quad\left(J_{m r}+N^{2}\left(\frac{m R_{1}^{2}}{4}+J_{w}+\frac{J R_{1}^{2}}{W^{2}}\right)\right) \frac{\mathrm{d} W_{m r}(t)}{\mathrm{d} t} \\
& +N^{2}\left(\frac{m R_{1}^{2}}{4}-\frac{J R_{1}^{2}}{W^{2}}\right) \frac{\mathrm{d} W_{m l}(t)}{\mathrm{d} t}
\end{aligned}
$$

TABLE I

Variables of the EPW

Using Eqs. (5) and (6) the system function block diagrams were obtained in s-domain, which are given in Fig. 2.

$J_{a a}$ and $J_{b b}$ in Fig. 2 are defined as given below:

$$
\begin{aligned}
& J_{a a}=N\left(\frac{m R_{1}^{2}}{4}+J_{w}+\frac{J R_{1}^{2}}{w^{2}}\right), \\
& J_{b b}=N\left(\frac{m R_{1}^{2}}{4}-\frac{J R_{1}^{2}}{w^{2}}\right) .
\end{aligned}
$$

From the general state space representation

$$
\begin{aligned}
& \dot{x}(t)=A x(t)+B u(t)+W d(t), \\
& y(t)=C x(t),
\end{aligned}
$$

the continuous time state space model of the EPW is directly obtained from the Eq. (5) and Eq. (6). The state space model is written in vector-matrix form, shown below.

$$
\begin{aligned}
& {\left[\begin{array}{c}
\frac{\mathrm{d} w_{m r}(t)}{\mathrm{d} t} \\
\frac{\mathrm{d} i_{a r}(t)}{\mathrm{d} t} \\
\frac{\mathrm{d} w_{m l}(t)}{\mathrm{d} t} \\
\frac{\mathrm{d} i_{a l}(t)}{\mathrm{d} t}
\end{array}\right]=\underbrace{\left[\begin{array}{cccc}
-J_{r r} B_{m r} & K_{t r} J_{r r} & -J_{r l} B_{m l} & K_{t l} J_{r l} \\
-\frac{K_{b r}}{L_{a r}} & -\frac{R_{a r}}{L_{a r}} & 0 & 0 \\
-J_{l r} B_{m l} & K_{t r} J_{l r} & -J_{l l} B_{m l} & K_{t l} J_{l l} \\
0 & 0 & -\frac{K_{b l}}{L_{a l}} & -\frac{R_{a l}}{L_{a l}}
\end{array}\right]}_{A}} \\
& \times\left[\begin{array}{c}
w_{m r}(t) \\
i_{a r}(t) \\
w_{m l}(t) \\
i_{a l}(t)
\end{array}\right]+\underbrace{\left[\begin{array}{cc}
0 & 0 \\
\frac{1}{L_{a r}} & 0 \\
0 & 0 \\
0 & \frac{1}{L_{a l}}
\end{array}\right]}_{B}\left[\begin{array}{l}
u_{a r}(t) \\
u_{a l}(t)
\end{array}\right]
\end{aligned}
$$




$$
-\underbrace{\left[\begin{array}{cc}
-N J_{r r} & N J_{r l} \\
0 & 0 \\
N J_{l r} & N J_{l l} \\
0 & 0
\end{array}\right]}_{W}\left[\begin{array}{c}
T_{\text {Load } r}(t) \\
T_{\text {Load } l}(t)
\end{array}\right] .
$$

The output matrix $C$ is given in Eq. (11):

$$
\left[\begin{array}{l}
y_{1}(t) \\
y_{2}(t)
\end{array}\right]=\underbrace{\left[\begin{array}{cccc}
N & 0 & 0 & 0 \\
0 & 0 & N & 0
\end{array}\right]}_{C}\left[\begin{array}{c}
w_{m r}(t) \\
i_{a r}(t) \\
w_{m l}(t) \\
i_{a l}(t)
\end{array}\right] .
$$

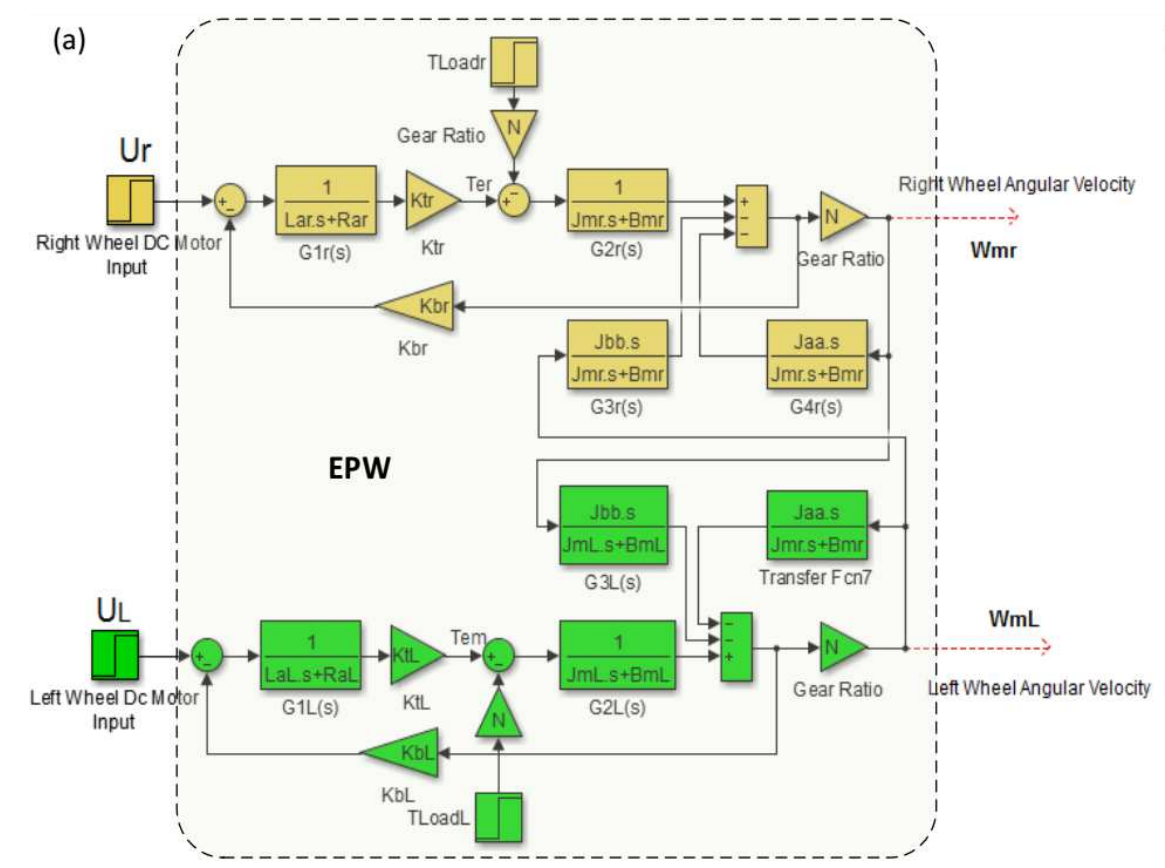

(b)

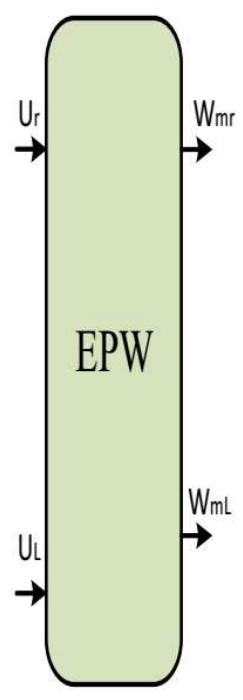

Fig. 2. (a) Transfer function blocks of EPW. (b) Input-output block diagram of EPW.

Luenberger observer is designed in discrete time, for this purpose first, state space model must be transformed from continuous to discrete time. The sampling time is selected as $T=\left(L_{a} / R_{a}\right) / 5$ by considering $L_{a}=L_{a r}=$ $L_{a l}, R_{a}=R_{a r}=R_{a l}$. The disturbance effect $(W=0)$ is neglected while designing the observer. Discretization steps are described in the next section.

\subsection{Discretization of continuous time state space model}

The continuous-time state space model in Eq. (6) can be discretized, assuming zero-order holds for the input $u$, thus Eq. (12) is obtained:

$$
\begin{aligned}
& x[k+1]=G x[k]+H u[k], \\
& y[k]=C_{d} x[k] .
\end{aligned}
$$

The discrete time matrices $G$ and $H$ can be calculated using Eqs. (13) and (14) [10], where $T$ is sampling time,

$$
\begin{aligned}
G & =\left.\mathrm{e}^{A t}\right|_{t=T}, \\
H & =\left(\int_{\tau=0}^{T} \mathrm{e}^{A \tau} \mathrm{d} \tau\right) B .
\end{aligned}
$$

\subsection{Luenberger observer and design for EPW}

The model of observer used in this study estimates the four state variables which are velocity of the right and left wheels of EPW $\left(W_{m r}, W_{m l}\right)$ and right and left DC motor currents $\left(i_{a r}, i_{a l}\right)$. Luenberger observer [6], is defined by the Eq. (15).

$$
\hat{x}(k+1)=\underbrace{A \hat{x}(k)+B u(k)}_{\text {Estimator }}+L[\underbrace{y(k)-C \hat{x}(k)}_{\text {Regulator }}] .
$$

The state variables observed by Luenberger observer are denoted by $\hat{x}(k)$ in Eq. (15). The observer is said to be asymptotically stable if the observer error defined by $e(k)=x(k)-\hat{x}(k)$ converges to zero when $k \rightarrow \infty$. The error dynamics model of the Luenberger observer is $e(k+1)=(A-L C) e(k) . L$ matrix is calculated using Ackerman formula and the poles of the observer $A-L C$ are chosen to converge five times faster than the fastest time constant of the system.

\section{Simulation study}

Simulation results are organized in two subsections. In the first one the comparison of continuous time state space model given in Eq. (10) with continuous time transfer functions block, depicted in Fig. 2, is presented. In the second subsection, the designed observer outputs are compared with the real measurement state variables for validation. All simulations are performed in MATLAB/Simulink environment. Block diagram of the simulation study is shown in Fig. 3. 


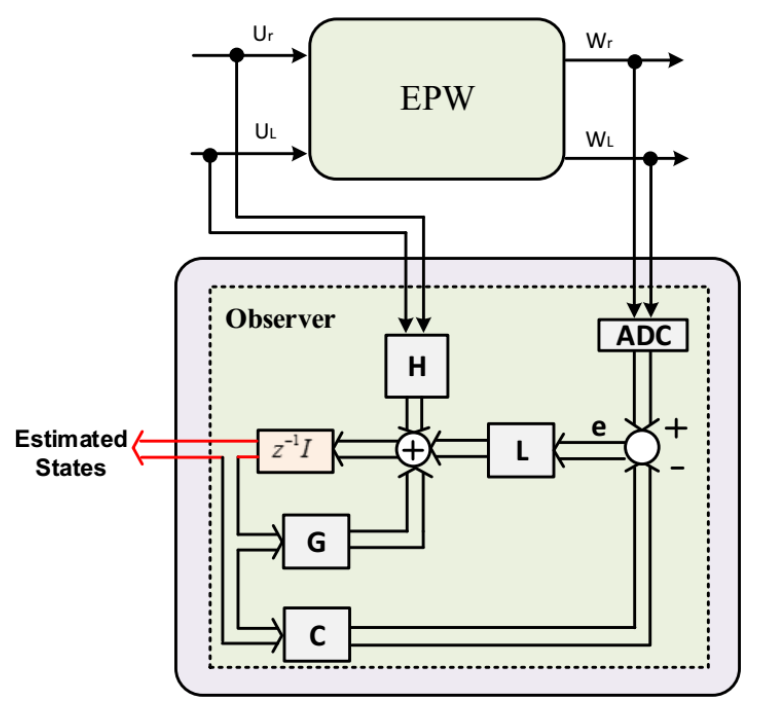

Fig. 3. Block diagram of simulation study.

TABLE II

Parameters used in experiment.

\begin{tabular}{|c|c|c|c|}
\hline Symbol & Value & Unit & Description \\
\hline$m$ & 90 & {$[\mathrm{~kg}]$} & $\begin{array}{l}\text { Total mass of the wheelchair body } \\
\text { and person on board }\end{array}$ \\
\hline$R_{1}$ & 0.305 & {$[\mathrm{~m}]$} & Radius of rear wheels \\
\hline$J_{w}$ & 0.017 & {$\left[\mathrm{Kg} \mathrm{m}^{2} / \mathrm{rad}\right]$} & Moment of inertia of the rear wheel \\
\hline$J$ & 0.23 & {$\left[\mathrm{Kg} \mathrm{m}^{2} / \mathrm{rad}\right]$} & $\begin{array}{l}\text { Total moment of inertia } \\
\text { with the total mass }\end{array}$ \\
\hline$L$ & 0.53 & {$[\mathrm{~m}]$} & Distance between rear wheels \\
\hline$L_{a}$ & 0.0016 & {$[\mathrm{H}]$} & DC motor inductance \\
\hline$R_{a}$ & 0.34 & {$[\Omega]$} & DC motor resistance \\
\hline$K_{t}$ & 0.17 & {$[\mathrm{~N} \mathrm{~m} / \mathrm{A}]$} & Torque constant of the DC motor \\
\hline$K_{b}$ & 0.17 & {$[\mathrm{Vs} / \mathrm{rad}]$} & $\begin{array}{l}\text { Reverse electromagnetic force } \\
\text { constant of the DC motor }\end{array}$ \\
\hline$N$ & 0.724 & & Gear ratio \\
\hline$B_{m}$ & 0 & {$[\mathrm{Nms}]$} & Motor viscous friction constant \\
\hline$J_{m}$ & 0.0011 & {$\left[\mathrm{Kg} \mathrm{m}^{2} / \mathrm{rad}\right]$} & Moment of inertia of the rotor \\
\hline$T$ & 0.00094 & [ms] & Sampling time \\
\hline
\end{tabular}

Using the parameters from Table II and Eqs. (13) and (14), the discrete time state space model matrices $G$ and $H$ are calculated as follows:

$$
\begin{aligned}
& G=\left[\begin{array}{cccc}
1 & 0.0008 & 0 & -0.0008 \\
-0.0906 & 0.8187 & 0 & 0 \\
0 & -0.0008 & 1 & 0.0008 \\
0 & 0 & -0.0906 & 0.8187
\end{array}\right], \\
& H=\left[\begin{array}{cc}
0.0003 & -0.0002 \\
0.533 & 0 \\
-0.0002 & 0.0003 \\
0 & 0.533
\end{array}\right] .
\end{aligned}
$$

The calculated observer gain matrix $L$ is given in Eq. (17) below:

$$
L=\left[\begin{array}{cc}
0.002 & 0 \\
0.427 & 4.08 \\
0 & 0.002 \\
4.08 & 4.427
\end{array}\right]
$$

Discrete time observer state space model is obtained in Eq. (18), where $\hat{w}_{m r}=\hat{x}_{1}(k), \hat{i}_{a r}=\hat{x}_{2}(k), \hat{w}_{m L}=\hat{x}_{3}(k)$, $\hat{i}_{a L}=\hat{x}_{4}(k)$.

$$
\begin{gathered}
{\left[\begin{array}{l}
\hat{x}_{1}(k+1) \\
\hat{x}_{2}(k+1) \\
\hat{x}_{3}(k+1) \\
\hat{x}_{4}(k+1)
\end{array}\right]=\left[\begin{array}{cccc}
1 & 0.0008 & 0 & -0.0008 \\
-0.0906 & 0.8187 & 0 & 0 \\
0 & -0.0008 & 1 & 0.0008 \\
0 & 0 & -0.0906 & 0.8187
\end{array}\right]} \\
\times\left[\begin{array}{l}
\hat{x}_{1}(k) \\
\hat{x}_{2}(k) \\
\hat{x}_{3}(k) \\
\hat{x}_{4}(k)
\end{array}\right]+\left[\begin{array}{cc}
0.0003 & -0.0002 \\
0.53 & 0 \\
-0.0002 & 0.0003 \\
0 & 0.53
\end{array}\right] u(k)+\left[\begin{array}{cc}
0.002 & 0 \\
0.427 & 4.08 \\
0 & 0.002 \\
4.08 & 4.427
\end{array}\right] \\
\times\left[y(k)-\left[\begin{array}{ccc}
0.72 & 0 & 0 \\
0 & 0 & 0.72
\end{array}\right]\left[\begin{array}{l}
\hat{x}_{1}(k) \\
\hat{x}_{2}(k) \\
\hat{x}_{3}(k) \\
\hat{x}_{4}(k)
\end{array}\right]\right]
\end{gathered}
$$

\subsection{Validation of state space model}

The EPW state space models and transfer function control block diagrams were obtained in Sect. 2 and the discrete time $G$ and $H$ matrices were calculated. The discrete time state space model must be validated before observer design. For this purpose, by comparing the response of continuous time state space model with the response of Laplace transfer functions block diagram, the accuracy of the model used for observer design is validated. Simulation setup is depicted in Fig. 4 and the results are shown in Fig. $5 \mathrm{a}$ and b respectively for step inputs.

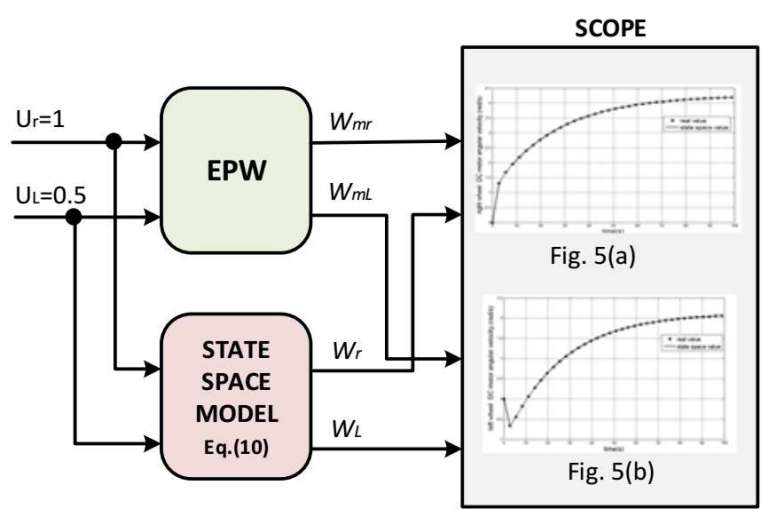

Fig. 4. Simulation study for state space validation.

Figure 5a and b shows that angular velocity of the right wheel $w_{r}(t)$ and left wheel $w_{l}(t)$, which are the state outputs of EPW, are equal to Laplace transform model angular velocity of right and left wheel $\left(W_{m r}, W_{m l}\right)$. The results show that the state space model is reliable for using the observer design. 

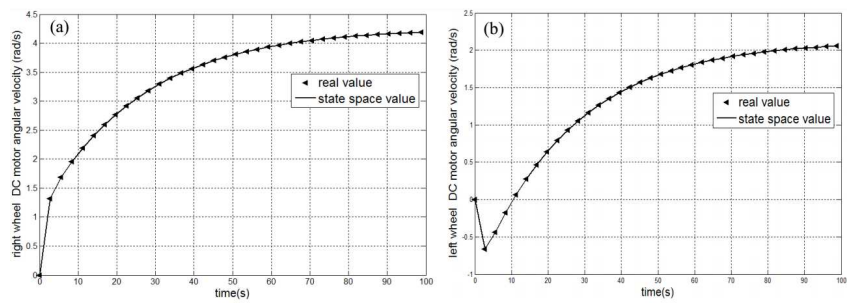

Fig. 5. Result of real angular velocity and state space model angular velocity, (a) right wheel with $U_{r}=1$, (b) left wheel with $U_{l}=0.5$.

\subsection{Observer simulation results}

The Luenberger observer was designed in Sect. 2. The discrete time observer model given in Eq. (18) was simulated in MATLAB/Simulink and compared with real model outputs. The results are shown in Fig. 6a and b, respectively.
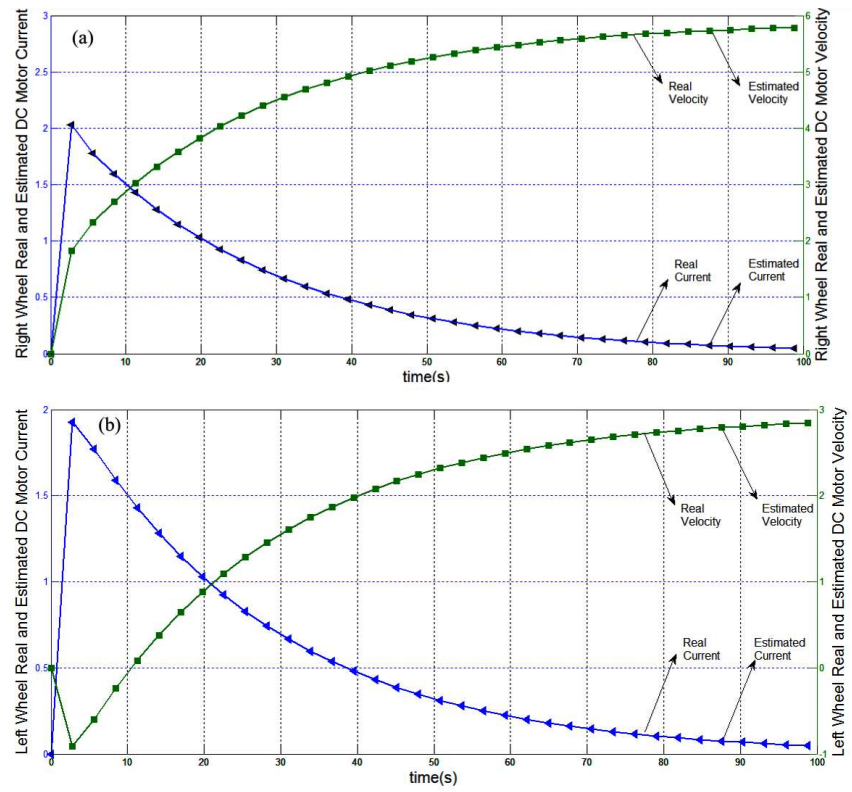

Fig. 6. Simulation results of DC motor real current and estimated DC motor current and velocity, (a) right wheel with $U_{r}=1$, (b) left wheel with $U_{l}=0.5$.

Figure $6 \mathrm{a}$ and $\mathrm{b}$ shows that estimated right $i_{a r}(t)$ and left $i_{a l}(t)$ DC motor currents and the real currents are identical. Furthermore, the estimated right and left wheel DC motor angular velocities $\left(W_{r}, W_{l}\right)$ are equal to real values $\left(W_{m r}, W_{m l}\right)$. Therefore, the observer estimates the DC motor state variables with a high accuracy.

\section{Conclusions}

Although in literature, model of the EPW is generally obtained from approximate models or generalized transfer functions, as a contribution to the literature, in this paper a discrete time state space model of the EPW is obtained directly from dynamic equations of the EPW. The simulation results show that the discrete time state space model is reliable for observer design and the Luenberger observer estimates the left and right wheel DC motor currents and velocities with a high accuracy.

\section{References}

[1] N. Louis, P. Gorce, in: IECON 2012 - 38th Annual Conference on IEEE Industrial Electronics Society, Montreal, IEEE, 2012, p. 5562.

[2] A. Widyotriatmo, in: Control, Systems $\&$ Industrial Informatics (ICCSII), 2012 IEEE Conference on, IEEE, Bandung 2012, p. 64.

[3] M. Mazo, F.J. Rodríguez, J.L. Lázaro, J. Ureńa, J.C. García, E. Santiso, P. Revenga, Control Eng. Pract. 3, 665 (1995).

[4] C. De La Cruz, T.F. Bastos, R. Carelli, Control Eng. Pract. 19, 113 (2011).

[5] J.B. Shung, G. Stout, M. Tomizuka, D.M. Auslander, J. Dyn. Syst. Meas. Control 105, 101 (1983).

[6] C.C. Ou, C.J. Chen, T.C. Chen, 3CA 2010-2010 Int. Symp. Comput. Commun. Control Autom., 2010, Vol. 2, p. 63.

[7] M.L. Corradini, G. Orlando, J. Robot. Syst. 18, 317 (2001).

[8] D. Ding, R. Cooper, IEEE Control Syst. Mag. 25, 22 (2005).

[9] M.N. Saadatzi, J. Poshtan, Proc. IEEE Int. Conf. Control Appl. 1, 1246 (2010).

[10] K. Ogata, Discrete-Time Control System, PrenticeHall, New Jersey 1995. 